\title{
Integrating molecular pathogenesis and clinical translation in sepsis-induced acute respiratory distress syndrome
}

\author{
Joshua A. Englert, ${ }^{1}$ Christopher Bobba, ${ }^{1,2}$ and Rebecca M. Baron ${ }^{3}$ \\ 'Division of Pulmonary, Critical Care and Sleep Medicine, The Ohio State University Wexner Medical Center, Columbus, \\ Ohio, USA. ${ }^{2}$ Department of Biomedical Engineering, The Ohio State University, Columbus, Ohio, USA. ${ }^{3}$ Division of \\ Pulmonary and Critical Care Medicine, Brigham and Women's Hospital, Boston, Massachusetts, USA.
}

Sepsis-induced acute respiratory distress syndrome (ARDS) has high morbidity and mortality and arises after lung infection or infection at extrapulmonary sites. An aberrant host response to infection leads to disruption of the pulmonary alveolar-capillary barrier, resulting in lung injury characterized by hypoxemia, inflammation, and noncardiogenic pulmonary edema. Despite increased understanding of the molecular biology underlying sepsis-induced ARDS, there are no targeted pharmacologic therapies for this devastating condition. Here, we review the molecular underpinnings of sepsis-induced ARDS with a focus on relevant clinical and translational studies that point toward novel therapeutic strategies.

Conflict of interest: The authors have declared that no conflict of interest exists.

Published: January 24, 2019

\section{Reference information:} JCI Insight. 2019;4(2):e124061. https://doi.org/10.1172/jci. insight.124061.

\section{Introduction}

Sepsis is a syndrome characterized by organ dysfunction and an aberrant immune response to an infection. Organ dysfunction results from the local or systemic release of mediators that exert deleterious effects at the site of infection or in organs distant from the inciting infection (1). Lung injury is common in sepsis, and when this occurs it results in acute respiratory distress syndrome (ARDS). Therapies to prevent or treat lung injury in sepsis remain elusive; therefore, it is critical to understand the molecular mechanisms that lead to sepsis-induced ARDS and the translational implications of these findings.

The 2016 Sepsis-3 conference (2) defined sepsis as life-threatening organ dysfunction caused by a dysregulated host response to infection. Clinical sepsis criteria were refined to include an acute change of greater than or equal to 2 points in the Sequential Organ Failure Assessment score, which assigns points for markers of injury to various organ systems (3). Septic shock was defined as sepsis resulting in an elevated blood lactate level ( $>2 \mu \mathrm{mol} / \mathrm{L})$ and requiring vasopressors to maintain adequate blood pressure (mean arterial pressure $\geq 65 \mathrm{mmHg}$ ) in the absence of hypovolemia. Patients with septic shock had significantly higher mortality than those with sepsis alone ( $>40 \%$ vs. $>10 \%$ ). Importantly, aspects of this definition are still undergoing critical evaluation (4-9). ARDS is defined by the acute (less than 7 days) onset of hypoxemia and bilateral radiographic infiltrates consistent with pulmonary edema that are not explained by heart failure (10). ARDS severity is determined by the degree of hypoxemia, as measured by the ratio of the partial pressure of oxygen in the blood $\left(\mathrm{PaO}_{2}\right)$ to the fraction of inspired oxygen delivered $\left(\mathrm{FIO}_{2}\right)$, with a lower $\mathrm{PaO}_{2} / \mathrm{FIO}_{2}$ ratio indicating more severe lung injury. The mortality rate for patients with severe ARDS $\left(\mathrm{PaO}_{2} / \mathrm{FIO}_{2}<100\right)$ approaches $40 \%$, with an intensive care unit (ICU) prevalence of $10 \%$, affecting nearly 1 in 4 mechanically ventilated patients (11). In a recent international study, sepsis was an underlying cause for approximately $75 \%$ of patients with ARDS (59\% pneumonia, 16\% extrapulmonary sepsis) (11), and it is estimated that there are over 210,000 cases of sepsis-induced ARDS in the US annually $(12,13)$. Notably, patients with sepsis-induced ARDS have higher mortality than those with ARDS from other causes (14). There may be unique sepsis-activated molecular pathways that result in ARDS and are distinct from those activated by other causes of ARDS (e.g., trauma, multiple transfusions). For example, certain pathways discussed below, such as pyroptosis or downstream effectors of mesenchymal stromal cells and pro-resolving lipid mediators, appear to enhance bacterial clearance, suggesting a more specific role in sepsis-induced ARDS. Additionally, studies have suggested that biomarkers correlating with higher levels of inflammation (e.g., procalcitonin, soluble ICAM-1, soluble E-selectin) and endothelial dysfunction (e.g., vWF antigen 
and soluble urokinase-type plasminogen activator receptor) might be enhanced in sepsis-induced ARDS compared with other causes of ARDS (15-17). Phenotyping ARDS patients based on biology underlying the development of lung injury has been an intense focus of study in recent years. In fact, some experts felt that clinical biomarkers should have been incorporated into consensus conference definitions, thus adding urgency to the quest for improved correlation of molecular pathways with clinical phenotypes (18).

Below, we highlight aspects of the current understanding of sepsis-induced ARDS that have led to translational studies and clinical trials targeting the molecular pathogenesis of lung injury following infection.

\section{Pathophysiology}

The gas-exchanging unit of the lung, the alveolus, is lined by a thin (several microns thick) alveolar-capillary barrier that maintains the air-liquid interface (Figure 1). The barrier has 3 components: (1) epithelial cell layer (either type I [AT1] or type II [AT2] pneumocytes), (2) microvascular endothelial cell layer, and (3) interstitial space between the epithelial and endothelial surfaces. Resident alveolar macrophages sit directly on top of pulmonary epithelia. The central concept that defines lung injury in ARDS is loss of this barrier (19). Sepsis-induced injury can initiate on the epithelial side (direct lung injury) or the endothelial side (indirect lung injury) (Figure 1). Barrier dysfunction from sepsis-induced ARDS can arise from an infection originating in the lung (e.g., pneumonia) or from extrapulmonary infection (e.g., intraabdominal infection) (Figure 1). The lung has approximately $480 \times 10^{6}$ alveoli (20) that can be differentially affected when ARDS develops, resulting in substantial heterogeneity, with severe injury to some areas of the lung and relative sparing of other areas.

In direct sepsis-induced ARDS, lung dysfunction begins following pneumonia. Pulmonary infections in adults were believed to be most commonly bacterial; however, recent evidence supports an increasing incidence of viral infections or combined bacterial/viral infections (21). Immunocompromised patients may be at increased risk for fungal or other atypical infections. Immune suppression or underlying lung pathology (e.g., interstitial lung disease; ref. 22) may increase risk for developing ARDS from pneumonia, but factors that predict sepsis-induced ARDS development in patients with pneumonia have not been clearly identified. Pulmonary pathogens activate a robust innate immune response in epithelial cells and alveolar macrophages, followed by neutrophil infiltration and monocyte recruitment that contribute to sepsis-induced ARDS (23, 24). Release of proinflammatory mediators, such as TNF, IL-1 $\beta$, and IL-6, lead to loss of alveolar-capillary barrier integrity, neutrophil recruitment, surfactant dysfunction, and alveolar edema (Figure 1). Changes in alveolar epithelial cells during sepsis-induced ARDS include alterations in alveolar fluid clearance, cell-cell junction formation, and cell trauma or death (25-27). Pathways downstream of epithelial injury (discussed below) may be disproportionately activated following direct lung injury. This concept is supported by the observation that biomarkers related to epithelial dysfunction (e.g., surfactant protein D) are elevated in ARDS patients with direct lung injury, compared with those with indirect lung injury (28).

Indirect sepsis-induced ARDS arises from an infection outside the lung. The mechanism(s) underlying the development of lung injury from an infection at a distant site are multifactorial and not well understood. The gut plays an important role in indirect lung injury, either as a primary source of infection or as the result of compromised gut barrier integrity due to infection at another site (29). Increased gut permeability can lead to the release of mediators that play a role in the development of lung injury (Figure 1). Toxic mediators released from the gut may also travel to the lung through mesenteric lymphatics, known as the "gut-lymph" hypothesis (30). The highly vascular nature of the lung and the fact that the entire cardiac output circulates through the pulmonary circulation predisposes this organ to injury. Damage to the alveolar-capillary barrier can be further fueled by alterations to microvascular endothelial cells and through formation of microthrombi. Injury to the endothelial cell surface, through alteration of the glycocalyx, formation of platelet-leukocyte aggregates, and triggered release of inflammatory mediators, can also facilitate loss of alveolar-capillary barrier function (31-33). Thus, in contrast with direct lung injury, pathways downstream of endothelial injury (discussed below) may be disproportionately activated following indirect lung injury. This concept is supported by the observation that biomarkers related to endothelial dysfunction (e.g., angiopoietin-1) are elevated in ARDS patients with indirect lung injury, compared with those with direct lung injury $(28,34)$.

Patients with sepsis-induced ARDS often require mechanical ventilation. Although lifesaving, mechanical ventilation can exacerbate underlying lung injury through a process known as ventilator-induced lung injury (VILI) $(35,36)$. Heterogeneity of lung injury during sepsis-induced ARDS predisposes local regions to overdistension (volutrauma) or alveolar collapse and reopening (atelectrauma) during mechanical ven- 
Indirect Lung Injury

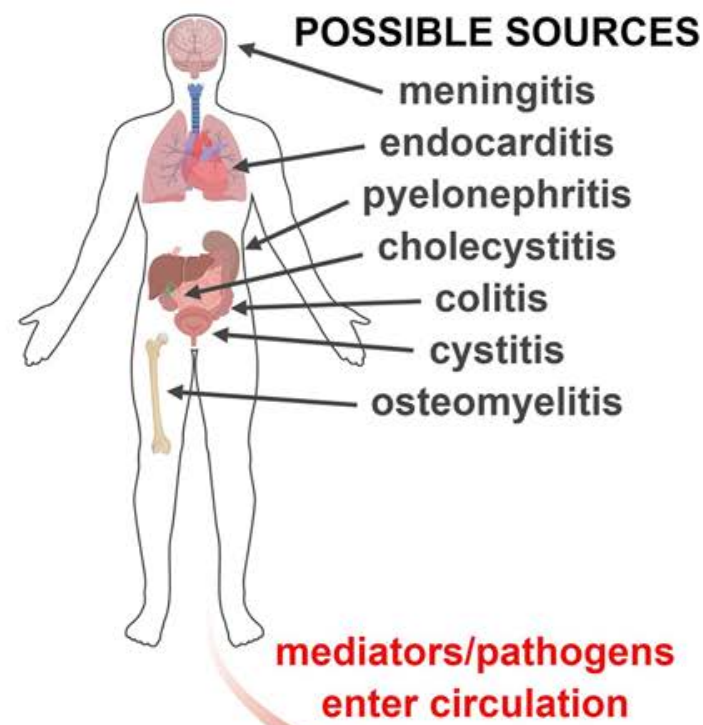

\section{Direct Lung Injury}
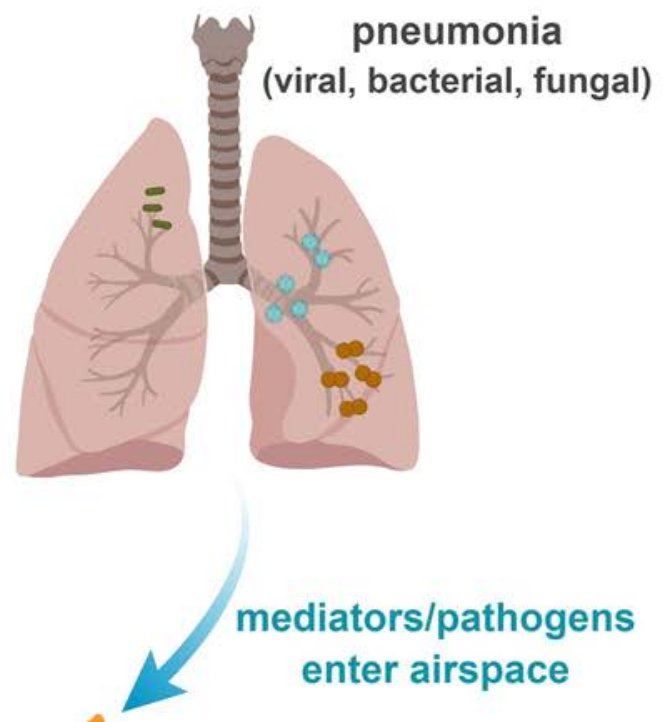

mediators/pathogens enter airspace
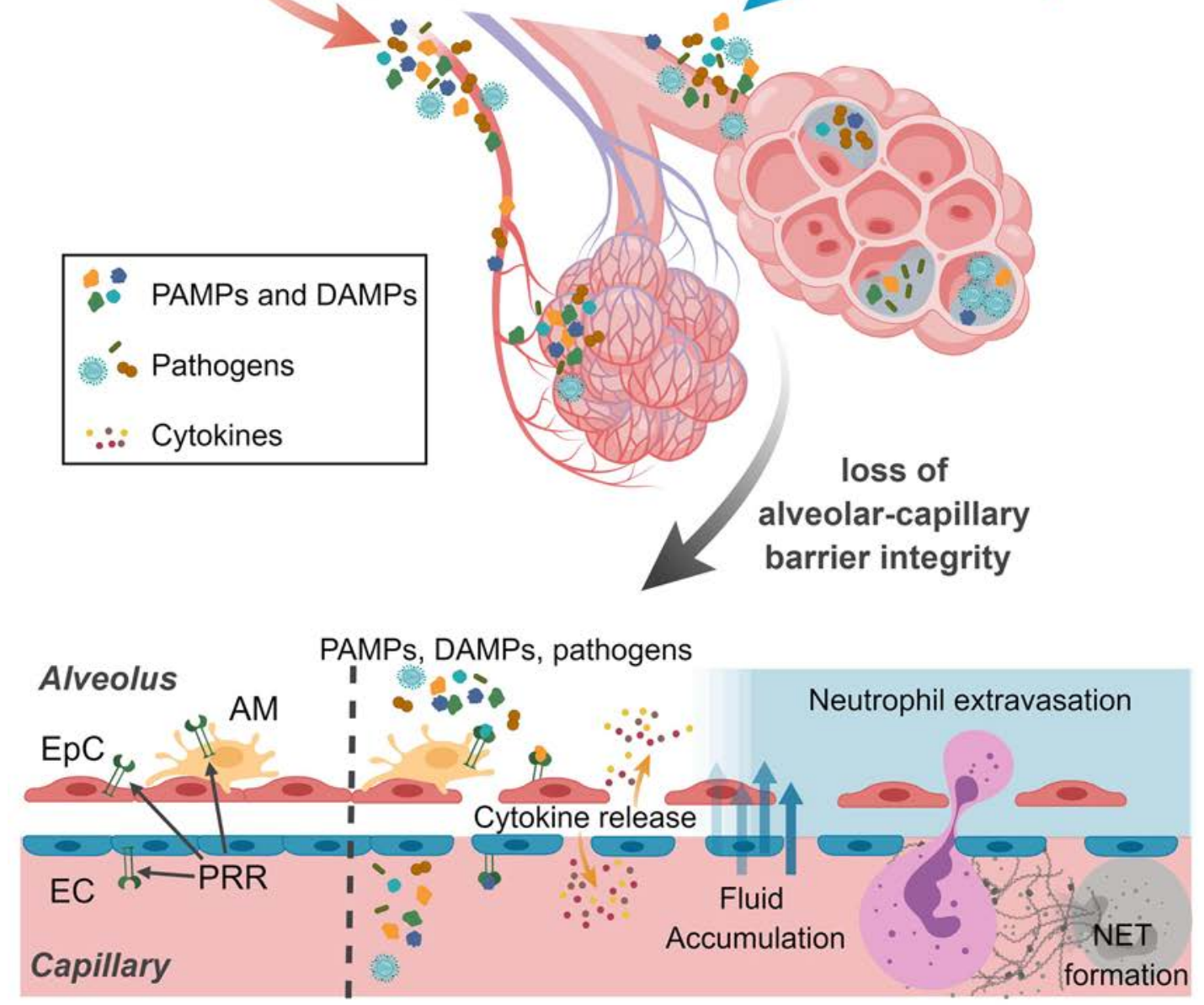

Normal Barrier

Sepsis Induced ARDS

Figure 1. Pathogenesis of sepsis-induced ARDS. Sepsis-induced ARDS arises from a lung infection (direct lung injury) or from an extrapulmonary source (indirect lung injury). The host response to the pathogen results in recruitment of inflammatory cells, release of proinflammatory cytokines, and other pathways of injury (see text) that damage the alveolar-capillary barrier. Loss of integrity of this barrier leads to influx of pulmonary edema fluid and lung injury. AM: alveolar macrophage, DAMPs: damage-associated molecular patterns, EC: endothelial cell, EpC: epithelial cell, NET: neutrophil extracellular trap, PAMPs: pathogen-associated molecular patterns, PRR: pattern recognition receptor.

tilation. Injurious mechanical forces can damage the lung through direct trauma to cells or through activation of mechanotransduction-associated biologic pathways (biotrauma). In preclinical models of sepsis-induced ARDS, mechanical ventilation produces synergistic injury compared with sepsis or VILI alone (37, 38). These are referred to as "two-hit" models and mirror the course of human sepsis (39). 


\section{Molecular pathways of lung injury}

Our current understanding of the biology underlying sepsis and ARDS is vast, and many detailed reviews have recently been published $(30,40-42)$. We focus on selected findings and contextualize them as they pertain to sepsis-induced ARDS.

Generation of inflammation. Sepsis-induced ARDS is initiated by an inflammatory host response to a microbial pathogen. The host response can be categorized into the innate immune response, which functions via pattern recognition receptors (PRRs) directed against microbial molecules and the adaptive immune response (humoral immunity from antibodies produced by B lymphocytes and cell-mediated immunity mediated by $\mathrm{T}$ lymphocytes), as well as host-derived molecules, leading to activation of cellular immune responses.

PRRs are membrane-bound or cytoplasmic proteins that bind to corresponding PAMPs, such as microbial nucleic acids, bacterial cell surface lipoproteins, and LPS. PRRs are present on surveillance cells, such as immune, epithelial, and endothelial cells, that sample the local environment (41). For example, Gram-negative bacteria-derived LPS is a PAMP that binds the cell-surface PRR TLR4, leading to innate immune response activation (43). The PRR family also includes transmembrane proteins, such as other TLRs and C-type lectin receptors, and cytoplasmic proteins, such as NOD-like receptors and retinoic acidinducible gene-I-like receptors (44). In addition to microbial signals, PRRs recognize endogenous DAMPs released from intracellular and extracellular compartments during sepsis. DAMPs include those of nuclear origin (e.g., DNA, high-mobility group box 1 protein) and those from the cytoplasm (e.g., RNA), the ECM (e.g., hyaluronic acid), or other sites (e.g., mitochondrial DNA [mtDNA], ATP) (42, 45-47). An initial septic insult produces downstream inflammation from PAMP stimulation of the innate immune system. This inflammation is subsequently amplified by DAMP signaling, resulting in further cell and tissue injury (41). These pathways trigger a cycle of injury, leading to alveolar-capillary barrier dysfunction, facilitation of additional pathogen invasion, and PAMP stimulation of the innate immune system.

Early control of infection provides the best defense against the release of PAMPs and DAMPs. Data from clinical studies support early antibiotics as a critical intervention for improving mortality from sepsis and development of end-organ dysfunction. Mortality from sepsis is 5 times higher when the initial antibiotic choice is inappropriate (48), and increased time to antibiotic administration is associated with progression to septic shock (49). Activation of the innate immune response following infection is regulated by various cellular pathways, including autophagy, a highly conserved pathway involved in clearing damaged proteins and organelles from cells. Following activation of the innate immune response, autophagy plays a key role in limiting mtDNA release, which is dependent upon the NALP3 inflammasome (50). Inflammasomes are multiprotein complexes that mediate caspase-1 activation, which promotes maturation and secretion of IL-1 $\beta$ and IL-18. Interestingly, mice lacking key autophagy proteins produced higher levels of IL-1 $\beta$ and IL-18 in sepsis models and had higher LPS- and polymicrobial sepsis-induced mortality than WT controls (51-53).

Our group and others have observed a striking association between elevated blood mtDNA levels and mortality in sepsis and ARDS, as well as an association between IL-18 levels and mortality (54-56). These data suggest that targeting inflammasome and autophagy pathways may represent a novel therapeutic strategy. LPS (via TLR4 stimulation) can also induce autophagy through a p38/MAPK/VPS34 pathway that results in activation of heme oxygenase-1 (HO-1), a cytoprotective molecule upregulated in sepsis and ARDS (57). CO, a gaseous molecule produced endogenously, is a byproduct derived from HO-1 induction $(58,59)$. Although toxic at high doses, low-dose $\mathrm{CO}$ administered exogenously is cytoprotective in preclinical sepsis and ARDS models (60). CO exerts its protective effect through pleiotropic mechanisms via regulation of autophagy and apoptosis, as well as through regulation of mitochondrial biogenesis and mesenchymal stem/stromal cells (MSCs) (57, 61-63). CO also enhances production and generation of pro-resolving lipid mediators (ref. 64 and see below). A recent multicenter phase I clinical trial examining the role of low-dose inhaled CO in sepsis-induced ARDS showed that low-dose inhaled CO administration in patients with sepsis-induced ARDS was feasible and safe, raising the prospect of a future targeted therapeutic to modify a DAMP-mediated injury cascade (65).

In addition to autophagy, programmed cell death plays an important role in lung inflammation and sepsis-induced lung injury. Previously, apoptosis, which is considered noninflammatory, was believed to be the only form of programmed cell death (66). More recently, other forms of programmed cell death have been reported, including necroptosis (necrotic cell death; ref. 67) and pyroptosis (68), both of which are proinflammatory. Pyroptosis is a caspase-1-dependent form of programmed cell death that occurs in response to infection with intracellular pathogens and is important for pathogen clearance. Immune cells recognize 
foreign pathogens, release proinflammatory cytokines, then "burst" and die, promoting tissue inflammation. Pyroptosis activated by the inflammasome can promote lung injury (69), including that caused by sepsis (51). Necroptosis is regulated by activation of the RIPK1-RIPK3 kinase complex pathway. Subsequent phosphorylation of the mixed-lineage kinase domain-like protein by receptor-interacting protein kinase 3 (RIPK3) activates necroptosis and leads to the release of cellular contents that propagate the inflammatory response (69). Many of the details of necroptosis signaling have been delineated in cancer cell lines, though necroptosis has recently been shown to contribute to VILI (70). RIPK3-deficient mice were protected from VILI and exhibited lower indices of cell necrosis compared with control animals. Furthermore, RIPK3 was found to be elevated in the plasma of mechanically ventilated patients, many of whom had sepsis-induced ARDS, demonstrating a role for this pathway in critically ill patients (70). In a subsequent study, elevated RIPK3 levels in plasma were associated with in-hospital mortality and organ failure in 5 ICU cohorts in the US and Korea (71). This work makes up part of a rapidly expanding network of nonapoptotic cell death pathways that may also contribute to organ dysfunction in sepsis-induced lung injury (67). Therapies that modulate these cell death pathways may limit inflammation or barrier disruption in the context of sepsis-induced ARDS, and cell death pathways may represent therapeutic targets in lung injury.

Dysregulated inflammation and downstream effectors. Dysregulation of the inflammatory response in sepsis and ARDS has been reviewed previously $(30,40,41)$. We will highlight some of the key findings, including recent progress using inflammatory profiles in patient phenotyping. Activation of the innate immune system by PAMPs or DAMPs leads to the release of numerous chemokines, as well as proinflammatory cytokines (e.g., IL-1 $\beta$, TNF, IL-6, IL-8) and antiinflammatory cytokines (e.g., IL-10) (30). Proinflammatory cytokines are important for pathogen clearance, but high levels can lead to alveolar-capillary barrier breakdown by injury to the endothelial layer (circulating cytokines) or injury to the epithelial layer (alveolar fluid cytokines) (72). After disruption of the alveolar-capillary barrier, proinflammatory effectors in the alveolar fluid are released into the circulation to promote further inflammation and immune responses. Notably, the use of lung-protective ventilation (i.e., low-tidal volume ventilation) has been shown to mitigate the release of various inflammatory cytokines (73).

Recently, it has become clear that there are subphenotypes of ARDS. Calfee et al. identified a hyperinflammatory subphenotype by applying latent class modeling to 2 cohorts from National Heart, Lung, and Blood Institute (NHLBI) ARDS randomized controlled trials (74). Compared with other ARDS patients, the hyperinflammatory subphenotype is characterized by higher plasma levels of proinflammatory biomarkers, including IL-8 and soluble TNF receptor 1; lower serum bicarbonate; and higher prevalence of sepsis. The hyperinflammatory group had worse outcomes, including higher mortality and fewer ventilator- and organ failure-free days. Moreover, patients with the hyperinflammatory subphenotype responded differently to the application of high positive end expiratory pressure on the ventilator, with a reduction in mortality that was not seen across the entire cohort (74). There may be additional subphenotypes of ARDS, such as a profibrotic subphenotype in which exacerbation of underlying interstitial lung abnormalities mimics ARDS (22).

Neutrophils are key effectors of the innate immune response to sepsis. These cells promote pathogen clearance by engulfing and killing bacteria and by releasing NETs, which are networks of extracellular DNA, including nuclear chromatin with associated proteins, that may facilitate pathogen clearance (Figure 1). However, delayed neutrophil apoptosis and prolonged neutrophilic inflammation can mediate further alveolar-capillary barrier disruption $(75,76)$. Interestingly, degradation of NETs with DNase1 improves lung injury and mortality in mouse models (77). Additionally, intravascular neutrophil and platelet activation can result in formation of neutrophil-platelet aggregates, leading to secondary capture of neutrophils, further promoting endothelial cell activation and barrier disruption. In preclinical ARDS models, blocking neutrophil-platelet aggregates mitigated the development of ARDS $(24,78)$. The complement system is also activated during sepsis, and PAMP or DAMP exposure leads to the production of peptides such as C3a and C5a. C5a is a potent proinflammatory peptide that functions as a chemoattractant for leukocytes and an amplifier of the inflammatory response. C5a triggers an oxidative burst in neutrophils, resulting in production of ROS and granular enzymes that contribute to tissue damage and barrier dysfunction (79). C5a can also promote NET formation (80).

Inflammatory network activation also affects the vascular and lymphatic endothelium, resulting in increased expression of adhesion molecules that facilitate binding of leukocytes to the endothelium. Severe sepsis can activate the coagulation system, with increased expression of procoagulant proteins (e.g., tissue factor, platelet activating factor) and a reduction in anticoagulant proteins (e.g., tissue factor pathway inhibitor, activated protein C). Fibrin removal is impeded, in part, by increased plasminogen activator inhibitor 
type 1 that inhibits the endogenous fibrinolytic system. As a result, increased microvascular clots can form that can promote tissue injury and end-organ dysfunction through impaired perfusion. In more severe cases of sepsis, widespread microvascular thrombosis can lead to disseminated intravascular coagulation and, paradoxically, thrombocytopenia and uncontrolled bleeding, likely from consumption of platelets and clotting factors. The coagulopathy of sepsis has been reviewed in detail previously (81).

Microvascular thrombosis and formation of platelet-leukocyte aggregates are likely key processes mediating sepsis-induced lung injury. Abdulnour et al. recently examined peripheral blood leukocyte number and activation in patients at risk for ARDS included in the Lung Injury Prevention Study with Aspirin trial of aspirin versus placebo (82), where aspirin did not reduce the risk of ARDS at 7 days. Many enrolled subjects had sepsis as their risk factor for ARDS development (>76\%). Unexpectedly, biomarkers of intravascular monocyte activation (i.e., monocyte-platelet aggregates) in at-risk patients were associated with ARDS development, pointing toward a role for activated monocytes in early ARDS pathogenesis (83). Future studies will need to address the functional implications of monocyte-platelet aggregates and monocyte subsets in lung injury development.

Immune suppression and immunoparalysis. Suppression of the adaptive immune response in sepsis may lead to a persistent compensatory antiinflammatory phase termed "immunoparalysis." Following initial cytokine-mediated hyperinflammation, immunoparalysis can lead to nosocomial infections and increased mortality in the later phase of sepsis (84). Although resuscitation strategies have improved early mortality in sepsis, patients often die in the later immunoparalysis phase, which is characterized by organ dysfunction, including the development of VILI in patients who require mechanical ventilation. Reactivation of viral infections, such as CMV, during this period (even in previously immunocompetent hosts) may confer additional risk for ARDS development (85). Strategies to prevent reactivation of latent viral infections is a potential approach to combat immunosuppression in sepsis. A recent phase II study examined whether ganciclovir in CMV-seropositive adults with sepsis-associated respiratory failure would prevent reactivation (86). There was no significant difference in the primary outcome (change in plasma IL-6 levels), but secondary analyses showed that ganciclovir decreased CMV reactivation and duration of mechanical ventilation and increased ventilator-free days. Although current data do not support the use of antiviral therapy to prevent reactivation or secondary infection, future studies will be needed to determine if this type of chemoprophylaxis can improve outcomes in the ICU.

Apoptosis-mediated depletion of $\mathrm{CD}^{+} \mathrm{T}$ cells and $\mathrm{B}$ cells has been proposed to lead to sepsis-induced immunoparalysis (87). Another potential mechanism is sepsis-related T cell exhaustion, during which dysfunctional $\mathrm{T}$ cells express programmed cell death protein 1 (PD-1) and cytotoxic T lymphocyte antigen- 4 (CTLA-4). Stromal and professional antigen-presenting cells increase expression of $\mathrm{T}$ cell programmed death ligand 1 (PD-L1) that binds PD-1 on T cells and suppresses $\mathrm{T}$ cell function (88). Furthermore, there is a shift toward a T helper type 2 immune-suppressive cytokine profile and increased activity of Tregs, which are also immune suppressive (88). Interestingly, T helper type 17 cells link the adaptive and innate immune systems, in part through IL-17 production and augmentation of neutrophil responses (89). Similar to cancer, reversal of $\mathrm{T}$ cell exhaustion has been proposed as a potential therapeutic approach in sepsis (90), and antibodies to CTLA-4, PD-1, and PD-L1 have shown benefit in murine sepsis models (91, 92). Other effectors of the immune system, including NKT cells, mucosal-associated invariant T cells, $\gamma \delta \mathrm{T}$ cells, CD ${ }^{+}$ T cells, and B cells, have previously been implicated in sepsis (see ref. 30 for detailed review).

Another potential strategy to prevent secondary infection and organ dysfunction during sepsis is to target immunodysfunction, with the goal of determining an individual's state of inflammation and/or immunoparalysis. This approach requires the ability to define the immunologic state of a patient with sepsis in real time and then administer treatment to alter that state. Such phenotyping would determine whether targeted therapy to suppress or boost the immune system could mitigate lung injury, and novel tools for rapid bedside immunophenotyping are being developed (93). Treatment with an antiinflammatory therapy may be appropriate for a patient in the hyperinflammatory phase of sepsis, while immunostimulatory therapies have a theoretical benefit during immunoparalysis. Examples of potential immunostimulatory therapies include leukocyte growth factors (GM-CSFs) that can increase neutrophil phagocytosis and killing (94); immunostimulatory cytokines (IFN- $\gamma$ and IL-7) that stimulate T cell survival and proliferation (95); and inhibitors of negative costimulatory pathways (PD-1 and PD-L1) (88). A recent study by Morrell et al. suggested that PD-L1 activation might hold promise for patients with sepsis-induced ARDS. The authors used cytometry by TOF (CyTOF) (96), combining flow cytometry with elemental mass spectrometry, to characterize immunophenotypic profiles of human alveolar leukocytes in ARDS (97). The majority of subjects with ARDS in this study had sepsis-induced ARDS, and 
expression of PD-1- and PD-L1-associated genes was significantly decreased in alveolar macrophages from ARDS subjects who required prolonged mechanical ventilation or died. The findings in this small cohort raise the interesting question as to whether PD-L1 expression on alveolar macrophages plays an important role in controlling the inflammatory response and whether activating PD-L1 may represent a novel therapeutic strategy in ARDS. Interestingly, PD-L1 checkpoint blockade in cancer patients has been associated with development of an ARDS-like pneumonitis (98), further supporting the relevance of this pathway in ARDS.

\section{Resolution and repair of injury}

Epithelial and endothelial cell repair. During sepsis-induced ARDS there is parallel activation of pathways that lead to lung injury and that promote resolution. The first step in the resolution of sepsis-induced lung injury is reestablishment of a functional alveolar-capillary barrier. On the epithelial side, this process begins with the migration and differentiation of AT2 cells to replace the injured AT1 cells, followed by proliferation of tissue resident progenitor cells. The release of soluble mediators from other structural lung cells plays a key role in alveolar epithelial repair. Keratinocyte growth factor (KGF) is released from fibroblasts and other lung cells and promotes epithelial repair (99). Administration of recombinant KGF or genetic overexpression decreases lung injury in preclinical models (100-102). However, a recent phase II trial of recombinant human KGF did not improve outcomes in ARDS and may have caused harm (103).

The lung endothelium must also be repaired during sepsis-induced ARDS. Endothelial permeability develops during lung injury through stimulation of the actin-myosin cytoskeleton and stress fiber formation in a Rho-dependent fashion (104). The release of inflammatory cytokines and other mediators of injury leads to the formation of intercellular gaps and leakage of edema fluid into the interstitial and alveolar spaces. Decreasing stress fiber formation by dampening Rho pathway activation leads to a compensatory activation of the Rap1 GTPase (105). Activation of Rap1 leads to restoration of adherens junctions and barrier integrity $(105,106)$. Another potent regulator of endothelial permeability is the bioactive lipid sphingosine-1-phosphate (S1P), which enhances barrier permeability through a series of signaling events that are initiated after binding to its membrane-bound GPCR (107). These pathways lead to the formation of focal adhesion kinases and the assembly of intercellular junctions (107). Interestingly, functional promoter variants in the S1P receptor S1PR3 were shown to associate with susceptibility to sepsis-induced ARDS (108).

Other molecules, including angiopoietin-1, have been shown to play key roles in maintenance and repair of the pulmonary endothelium (104). Angiopoietin-1 is a vascular growth factor that maintains capillary integrity via binding to the endothelial Tie2 receptor (109). Following vascular injury during sepsis-induced ARDS, angiopoietin-2 is released from endothelial cells and antagonizes angiopoietin-1 binding to Tie2, leading to increased vascular permeability (110). Angiopoietin-2 levels are increased in patients with ARDS and sepsis and are associated with worse outcomes (111-114). An angiopoietin-1 mimetic and angiopoietin-2-binding antibodies have been shown to prevent lung injury in preclinical ARDS (115) and sepsis (116) models and are currently being developed for possible use in patients with ARDS (117).

HMG CoA reductase inhibitors (i.e., statins) have also been investigated as a potential therapy in ARDS through protection of the endothelial barrier $(118,119)$. A recent report suggested that statins can also dampen inflammation from injurious mechanical forces during mechanical ventilation (120). Despite benefit in preclinical models, a randomized clinical trial of rosuvastatin versus placebo in sepsis-induced ARDS failed to show a difference in 60-day mortality and was stopped early for futility (121). A second randomized trial of simvastatin in ARDS (Hydroxymethylglutaryl-CoA reductase inhibition in Acute lung injury to Reduce Pulmonary dysfunction [HARP-2] trial) did not decrease ventilator-free days (122), but interestingly, a subsequent analysis of the HARP-2 data showed that patients with a hyperinflammatory subphenotype had increased survival when treated with simvastatin (123). These data highlight the importance of using a strategy to identify patients that are likely respond to a therapy (i.e., predictive enrichment) when conducting clinical trials in patients with ARDS (124).

Extracellular adenosine is an endogenous antiinflammatory mediator that decreases leukocyte recruitment to the lung (125) and enhances endothelial barrier function in the context of lung injury (126). In the vasculature, adenosine is generated from AMP by the endothelial enzyme ecto 5'-nucleotidase (CD73) (127). IFN- $\beta-1$ a increases CD73 expression in pulmonary capillaries, and a phase I/II trial of IFN- $\beta-1 \mathrm{a}$ in patients with ARDS demonstrated that this drug was safe and was associated with a decrease in 28-day mortality (128). However a recent phase III trial of IFN- $\beta-1 \mathrm{a}$ in ARDS did not show a significant improvement in mortality or ventilator-free days (129). 
The matrix, progenitor cells, and lung repair. Lung repair begins with the deposition of a provisional matrix to facilitate migration and proliferation of fibroblasts and other lung structural cells. The matrix also plays a key role in orchestrating the proliferation of various structural cells to restore normal lung architecture. Mesenchymal cells, including fibroblasts, produce collagen and other matrix components in response to the secretion of TGF- $\beta$ and other FGFs (130). Fibroblasts can also degrade and remodel the matrix through the secretion of metalloproteinases (131). Once lung structure and function have been reestablished, the provisional matrix is removed and fibroblasts return to a homeostatic and quiescent state. Failure to return to homeostasis results in persistent matrix deposition and fibroblast proliferation that is characteristic of the fibroproliferative phase of ARDS (132).

As the lung barrier is restored and fluid is cleared from alveoli, progenitor cells proliferate and differentiate to restore alveolar architecture and function. Although some controversy persists, the current consensus is that the adult lung contains multiple niches of progenitor cells that function as stem cells and proliferate in response to injury (133). Basal cells can self-renew and give rise to all cell types in the airway epithelium $(133,134)$. Recently, 2 groups found that activation of WNT signaling identified a subpopulation of AT2 cells responsible for proliferation and differentiation following epithelial injury from influenza (135) or hyperoxia (136). MSCs are found in many tissues and were originally defined by the ability to differentiate into multiple tissue types in vitro. Though they do not directly engraft or act as progenitor cells, MSCs possess immunomodulatory properties and decrease injury in preclinical ARDS models (137). A phase I trial of allogeneic bone marrow-derived MSCs for ARDS found that a single infusion of MSCs in patients with moderate to severe ARDS was well tolerated (138), which was confirmed in a recently published phase II trial (139).

Resolution of inflammation and clearance of inflammatory cells. Once a tight barrier has been reestablished, the next step is clearance of inflammatory cells, debris, and edema fluid from the alveolar and interstitial spaces. This process is mediated by decreased production and degradation of chemokines (140). As inflammation dampens, recruited neutrophils undergo apoptosis or other forms of cell death (see above). Apoptotic neutrophils are cleared through phagocytosis by macrophages and dendritic cells in a process known as efferocytosis, further decreasing the secretion of proinflammatory mediators (141) and increasing the secretion of antiinflammatory cytokines such as IL-10 (142). Alveolar macrophages and recruited monocytes are primarily responsible for removing apoptotic cells and other debris, such as pathogens, surfactant components, ECM components, and microparticles (143). Edema fluid is removed through active transport that is dependent on ion channels in the apical and basolateral membranes of lung epithelial cells. Epithelial sodium transport channels (ENaCs) on the surface of AT2 and AT1 cells permit passive movement of sodium from the airspaces into the cytoplasm (144). This gradient is maintained through active transport of sodium into the blood through the $\mathrm{Na} / \mathrm{K}$ ATPase in the basolateral membrane. Transport of water follows sodium from the alveolar space into the interstitial and plasma compartments for clearance. Alveolar fluid clearance (AFC) is regulated by changes in expression and membrane localization of both the ENaC and the $\mathrm{Na} / \mathrm{K}$ ATPase in preclinical models $(145,146)$ and is impaired in ARDS patients (147).

$\beta$-Adrenergic agonists enhance AFC by increasing membrane localization and expression of the $\mathrm{ENaC}$ and $\mathrm{Na} / \mathrm{K}$ ATPase channels in a cAMP-dependent manner (145). The NIH ARDS network albuterol for the treatment of acute lung injury (ALTA) trial examined the use of aerosolized albuterol to promote resolution of edema. The study was terminated for futility, with no significant difference in the primary endpoint (ventilator-free days) (148). A subsequent trial of intravenous salbutamol was also ineffective and was stopped early for increased mortality in the treatment group (149). Recently, the phase II Lung Injury Prevention Study with Budesonide and Beta Agonist, Formoterol, examined the early use of a combination of inhaled formoterol and budesonide in newly hypoxemic patients at risk for ARDS. Patients receiving the study drug had improved oxygenation with no significant adverse effects (150). Further study is needed to determine whether therapies that enhance AFC will be safe and efficacious in patients with ARDS, and specifically in patients with sepsis-induced ARDS.

The process of resolution and repair described above is orchestrated by different cell types and several classes of master regulators. Cells such as resident and recruited alveolar macrophages $(143,151)$ and Tregs (152) play a vital role in limiting injury and coordinating the repair process. These cells and others exert their effects through direct cell-cell interaction and through the release of secreted mediators. One class of mediators is specialized pro-resolving lipid mediators (SPMs), including lipoxins, resolvins, protectins, and maresins (153). These molecules coordinate a variety of cellular processes that dampen inflammation and promote restoration of the lung barrier by limiting leukocyte recruitment, decreasing cytokine release, promoting debris 
clearance from the alveolar space, and perhaps modulating pyroptosis $(153,154)$. Administration of SPMs improved outcomes in some preclinical ARDS models $(24,155)$, and a small study suggests that specific lipid mediator signatures in patients with sepsis might correlate with survival and predict ARDS development (156). Thus, SPMs hold promise as novel biomarkers and therapeutic targets in sepsis-induced ARDS.

\section{Conclusion}

Disruption of the alveolar-capillary barrier is the pathophysiologic hallmark of sepsis-induced ARDS. Initiation of the host response to infection and subsequent dysregulation of that response drives barrier disruption. Repair of the epithelium and endothelium are essential for resolution of sepsis-induced ARDS. When the repair process is impaired, lung injury can fail to resolve or progress to fibrosis. Developing effective therapies for sepsis-induced ARDS has proven difficult due to heterogeneity among ARDS patients, limitations of preclinical models, and a lack of readily available lung tissue from ARDS patients for research. Additionally, sepsis-induced ARDS can be initiated by a wide array of different inciting infections that might ultimately require different types of pathogen-specific treatment to interrupt lung injury. However, with increasing understanding of patient heterogeneity, recent discovery of novel biomarkers as potential therapeutic targets, and improved molecular tools for phenotyping patients, we remain optimistic that understanding the underlying biological processes will ultimately lead to the successful translation of new therapies to prevent or treat ARDS in patients with sepsis.

\section{Acknowledgments}

This work was supported by grants from National Institute of General Medical Sciences (R01GM115605 to RMB and K08GM102695 to JAE) and the NHLBI (R56HL142767 to JAE). JAE was also supported in part by grants from the American Thoracic Society Research Foundation and the Central Society for Clinical and Translational Research.

Address correspondence to: Joshua A. Englert, Division of Pulmonary, Critical Care, and Sleep Medicine, The Ohio State University Wexner Medical Center, 201 Davis Heart and Lung Research Institute, $473 \mathrm{~W}$ 12th Ave, Columbus, OH 43210, USA. Phone: 614.685.7038; Email: joshua.englert@osumc.edu.

1. Aziz M, Jacob A, Yang WL, Matsuda A, Wang P. Current trends in inflammatory and immunomodulatory mediators in sepsis. J Leukoc Biol. 2013;93(3):329-342.

2. Singer M, et al. The Third International Consensus Definitions for Sepsis and Septic Shock (Sepsis-3). JAMA. 2016;315(8):801-810.

3. Vincent JL, et al. The SOFA (Sepsis-related Organ Failure Assessment) score to describe organ dysfunction/failure. On behalf of the Working Group on Sepsis-Related Problems of the European Society of Intensive Care Medicine. Intensive Care Med. 1996;22(7):707-710

4. Raith EP, et al. Prognostic Accuracy of the SOFA Score, SIRS Criteria, and qSOFA Score for In-Hospital Mortality Among Adults With Suspected Infection Admitted to the Intensive Care Unit. JAMA. 2017;317(3):290-300.

5. Churpek MM, et al. Quick Sepsis-related Organ Failure Assessment, Systemic Inflammatory Response Syndrome, and Early Warning Scores for Detecting Clinical Deterioration in Infected Patients outside the Intensive Care Unit. Am J Respir Crit Care Med. 2017;195(7):906-911.

6. Bhattacharjee P, Edelson DP, Churpek MM. Identifying Patients With Sepsis on the Hospital Wards. Chest. 2017;151(4):898-907.

7. Marshall JC. Sepsis Definitions: A Work in Progress. Crit Care Clin. 2018;34(1):1-14.

8. Simpson SQ. SIRS in the Time of Sepsis-3. Chest. 2018;153(1):34-38.

9. Sterling SA, Puskarich MA, Glass AF, Guirgis F, Jones AE. The Impact of the Sepsis-3 Septic Shock Definition on Previously Defined Septic Shock Patients. Crit Care Med. 2017;45(9):1436-1442.

10. ARDS Definition Task Force, et al. Acute respiratory distress syndrome: the Berlin Definition. JAMA. 2012;307(23):2526-2533

11. Bellani G, et al. Epidemiology, patterns of care, and mortality for patients with acute respiratory distress syndrome in intensive care units in 50 countries. JAMA. 2016;315(8):788-800.

12. US and World Population Clock. US and World Population Estimates (2018). United States Census Bureau website. https:// www.census.gov/popclock/?intcmp=w_200x402. Accessed December 17, 2018.

13. Rubenfeld GD, et al. Incidence and outcomes of acute lung injury. N Engl J Med. 2005;353(16):1685-1693.

14. Stapleton RD, Wang BM, Hudson LD, Rubenfeld GD, Caldwell ES, Steinberg KP. Causes and timing of death in patients with ARDS. Chest. 2005;128(2):525-532.

15. Moss M, Gillespie MK, Ackerson L, Moore FA, Moore EE, Parsons PE. Endothelial cell activity varies in patients at risk for the adult respiratory distress syndrome. Crit Care Med. 1996;24(11):1782-1786.

16. Brunkhorst FM, Eberhard OK, Brunkhorst R. Discrimination of infectious and noninfectious causes of early acute respiratory distress syndrome by procalcitonin. Crit Care Med. 1999;27(10):2172-2176.

17. Wu X, Hu K, Yu L, Wang H, Long D. Correlation of plasma suPAR expression with disease risk and severity as well as prognosis of sepsis-induced acute respiratory distress syndrome. Int J Clin Exp Pathol. 2017;10(12):11378-11383. 
18. Abraham E. New definitions for sepsis and septic shock: continuing evolution but with much still to be done. JAMA. 2016;315(8):757-759.

19. Thompson BT, Chambers RC, Liu KD. Acute respiratory distress syndrome. N Engl J Med. 2017;377(6):562-572.

20. Ochs M, et al. The number of alveoli in the human lung. Am J Respir Crit Care Med. 2004;169(1):120-124.

21. Wunderink RG, Waterer G. Advances in the causes and management of community acquired pneumonia in adults. BMJ. 2017;358:j2471.

22. Putman RK, et al. Interstitial lung abnormalities are associated with acute respiratory distress syndrome. Am J Respir Crit Care Med. 2017;195(1):138-141.

23. Herold S, Becker C, Ridge KM, Budinger GR. Influenza virus-induced lung injury: pathogenesis and implications for treatment. Eur Respir J. 2015;45(5):1463-1478.

24. Abdulnour RE, et al. Maresin 1 biosynthesis during platelet-neutrophil interactions is organ-protective. Proc Natl Acad Sci U S A. 2014;111(46):16526-16531.

25. Schlingmann B, Molina SA, Koval M. Claudins: Gatekeepers of lung epithelial function. Semin Cell Dev Biol. 2015;42:47-57.

26. Matalon S, Bartoszewski R, Collawn JF. Role of epithelial sodium channels in the regulation of lung fluid homeostasis. Am J Physiol Lung Cell Mol Physiol. 2015;309(11):L1229-L1238.

27. Zeyed YF, Bastarache JA, Matthay MA, Ware LB. The severity of shock is associated with impaired rates of net alveolar fluid clearance in clinical acute lung injury. Am J Physiol Lung Cell Mol Physiol. 2012;303(6):L550-L555.

28. Calfee CS, et al. Distinct molecular phenotypes of direct vs indirect ARDS in single-center and multicenter studies. Chest. 2015;147(6):1539-1548.

29. Mittal R, Coopersmith CM. Redefining the gut as the motor of critical illness. Trends Mol Med. 2014;20(4):214-223.

30. McConnell KW, Coopersmith CM. Pathophysiology of septic shock: From bench to bedside. Presse Med. 2016;45(4 Pt 2):e93-e98.

31. Martin L, Koczera P, Zechendorf E, Schuerholz T. The endothelial glycocalyx: new diagnostic and therapeutic approaches in sepsis. Biomed Res Int. 2016;2016:3758278.

32. Colbert JF, Schmidt EP. Endothelial and microcirculatory function and dysfunction in sepsis. Clin Chest Med. 2016;37(2):263-275

33. Mehta D, Ravindran K, Kuebler WM. Novel regulators of endothelial barrier function. Am J Physiol Lung Cell Mol Physiol. 2014;307(12):L924-L935.

34. Murphy LS, et al. Endothelial glycocalyx degradation is more severe in patients with non-pulmonary sepsis compared to pulmonary sepsis and associates with risk of ARDS and other organ dysfunction. Ann Intensive Care. 2017;7(1):102.

35. Slutsky AS, Ranieri VM. Ventilator-induced lung injury. N Engl J Med. 2013;369(22):2126-2136.

36. Matthay MA, Ware LB, Zimmerman GA. The acute respiratory distress syndrome. J Clin Invest. 2012;122(8):2731-2740.

37. Villar J, Blanco J, Zhang H, Slutsky AS. Ventilator-induced lung injury and sepsis: two sides of the same coin? Minerva Anestesiol. 2011;77(6):647-653.

38. Zampieri FG, Mazza B. Mechanical Ventilation in Sepsis: A Reappraisal. Shock. 2017;47(1S suppl 1):41-46.

39. Baron RM, Choi AJ, Owen CA, Choi AM. Genetically manipulated mouse models of lung disease: potential and pitfalls. Am J Physiol Lung Cell Mol Physiol. 2012;302(6):L485-L497.

40. Wohlrab P, Kraft F, Tretter V, Ullrich R, Markstaller K, Klein KU. Recent advances in understanding acute respiratory distress syndrome. F1000Res. 2018;7:F1000 Faculty Rev-263.

41. Hotchkiss RS, Moldawer LL, Opal SM, Reinhart K, Turnbull IR, Vincent JL. Sepsis and septic shock. Nat Rev Dis Primers. 2016;2:16045.

42. Lewis AJ, Billiar TR, Rosengart MR. Biology and metabolism of sepsis: innate immunity, bioenergetics, and autophagy. Surg Infect (Larchmt). 2016;17(3):286-293.

43. Poltorak A, et al. Defective LPS signaling in $\mathrm{C} 3 \mathrm{H} / \mathrm{HeJ}$ and C57BL/10ScCr mice: mutations in Tlr4 gene. Science. 1998;282(5396):2085-2088.

44. Takeuchi O, Akira S. Pattern recognition receptors and inflammation. Cell. 2010;140(6):805-820.

45. Deutschman CS, Tracey KJ. Sepsis: current dogma and new perspectives. Immunity. 2014;40(4):463-475.

46. Iwasaki A, Medzhitov R. Regulation of adaptive immunity by the innate immune system. Science. 2010;327(5963):291-295.

47. Zhang Q, et al. Circulating mitochondrial DAMPs cause inflammatory responses to injury. Nature. 2010;464(7285):104-107.

48. Kumar A, et al. Initiation of inappropriate antimicrobial therapy results in a fivefold reduction of survival in human septic shock. Chest. 2009;136(5):1237-1248.

49. Whiles BB, Deis AS, Simpson SQ. Increased time to initial antimicrobial administration is associated with progression to septic shock in severe sepsis patients. Crit Care Med. 2017;45(4):623-629.

50. Nakahira K, et al. Autophagy proteins regulate innate immune responses by inhibiting the release of mitochondrial DNA mediated by the NALP3 inflammasome. Nat Immunol. 2011;12(3):222-230.

51. Lee S, et al. NLRP3 inflammasome deficiency protects against microbial sepsis via increased lipoxin B4 synthesis. Am J Respir Crit Care Med. 2017;196(6):713-726.

52. Moon JS, et al. NOX4-dependent fatty acid oxidation promotes NLRP3 inflammasome activation in macrophages. Nat Med. 2016;22(9):1002-1012.

53. Moon JS, et al. UCP2-induced fatty acid synthase promotes NLRP3 inflammasome activation during sepsis. J Clin Invest. 2015;125(2):665-680.

54. Dolinay T, et al. Inflammasome-regulated cytokines are critical mediators of acute lung injury. Am J Respir Crit Care Med. 2012;185(11):1225-1234.

55. Nakahira K, et al. Circulating mitochondrial DNA in patients in the ICU as a marker of mortality: derivation and validation. PLoS Med. 2013;10(12):e1001577.

56. Krychtiuk KA, et al. Mitochondrial DNA and Toll-like receptor-9 are associated with mortality in critically ill patients. Crit Care Med. 2015;43(12):2633-2641.

57. Carchman EH, Rao J, Loughran PA, Rosengart MR, Zuckerbraun BS. Heme oxygenase-1-mediated autophagy protects against hepatocyte cell death and hepatic injury from infection/sepsis in mice. Hepatology. 2011;53(6):2053-2062.

58. Chung SW, Liu X, Macias AA, Baron RM, Perrella MA. Heme oxygenase-1-derived carbon monoxide enhances the host 
defense response to microbial sepsis in mice. J Clin Invest. 2008;118(1):239-247.

59. Fredenburgh LE, et al. Absence of heme oxygenase-1 expression in the lung parenchyma exacerbates endotoxin-induced acute lung injury and decreases surfactant protein-B levels. Cell Mol Biol (Noisy-le-grand). 2005;51(5):513-520.

60. Ryter SW, Ma KC, Choi AMK. Carbon monoxide in lung cell physiology and disease. Am J Physiol Cell Physiol. 2018;314(2):C211-C227.

61. Lee S, et al. Carbon monoxide confers protection in sepsis by enhancing beclin 1-dependent autophagy and phagocytosis. Antioxid Redox Signal. 2014;20(3):432-442.

62. Suliman HB, Piantadosi CA. Mitochondrial biogenesis: regulation by endogenous gases during inflammation and organ stress. Curr Pharm Des. 2014;20(35):5653-5662.

63. Tsoyi $\mathrm{K}$, et al. Carbon monoxide improves efficacy of mesenchymal stromal cells during sepsis by production of specialized proresolving lipid mediators. Crit Care Med. 2016;44(12):e1236-e1245.

64. Dalli J, et al. The regulation of proresolving lipid mediator profiles in baboon pneumonia by inhaled carbon monoxide. Am $J$ Respir Cell Mol Biol. 2015;53(3):314-325.

65. Fredenburgh LE, et al. A phase I trial of low-dose inhaled carbon monoxide in sepsis-induced ARDS. JCI Insight. 2018;3(23):e124039.

66. Taylor RC, Cullen SP, Martin SJ. Apoptosis: controlled demolition at the cellular level. Nat Rev Mol Cell Biol. 2008;9(3):231-241

67. Vanden Berghe T, Linkermann A, Jouan-Lanhouet S, Walczak H, Vandenabeele P. Regulated necrosis: the expanding network of non-apoptotic cell death pathways. Nat Rev Mol Cell Biol. 2014;15(2):135-147.

68. Jorgensen I, Miao EA. Pyroptotic cell death defends against intracellular pathogens. Immunol Rev. 2015;265(1):130-142.

69. Fan EKY, Fan J. Regulation of alveolar macrophage death in acute lung inflammation. Respir Res. 2018;19(1):50.

70. Siempos II, et al. RIPK3 mediates pathogenesis of experimental ventilator-induced lung injury. JCI Insight. 2018;3(9):e97102.

71. Ma KC, et al. Circulating RIPK3 levels are associated with mortality and organ failure during critical illness. JCI Insight. 2018;3(13):e99692.

72. Huppert LA, Matthay MA. Alveolar fluid clearance in pathologically relevant conditions: in vitro and in vivo models of acute respiratory distress syndrome. Front Immunol. 2017;8:371

73. Curley GF, Laffey JG, Zhang H, Slutsky AS. Biotrauma and ventilator-induced lung injury: clinical implications. Chest. 2016;150(5):1109-1117.

74. Calfee CS, et al. Subphenotypes in acute respiratory distress syndrome: latent class analysis of data from two randomised controlled trials. Lancet Respir Med. 2014;2(8):611-620.

75. Frantzeskaki F, Armaganidis A, Orfanos SE. Immunothrombosis in acute respiratory distress syndrome: cross talks between inflammation and coagulation. Respiration. 2017;93(3):212-225.

76. Lefrançais E, Mallavia B, Zhuo H, Calfee CS, Looney MR. Maladaptive role of neutrophil extracellular traps in pathogen-induced lung injury. JCI Insight. 2018;3(3):e98178.

77. Caudrillier A, et al. Platelets induce neutrophil extracellular traps in transfusion-related acute lung injury. J Clin Invest. 2012;122(7):2661-2671.

78. Li R, et al. Maresin 1 mitigates inflammatory response and protects mice from sepsis. Mediators Inflamm. 2016;2016:3798465.

79. Ward PA. The harmful role of c5a on innate immunity in sepsis. J Innate Immun. 2010;2(5):439-445.

80. Garcia CC, et al. Complement C5 activation during influenza A infection in mice contributes to neutrophil recruitment and lung injury. PLoS One. 2013;8(5):e64443.

81. Simmons J, Pittet JF. The coagulopathy of acute sepsis. Curr Opin Anaesthesiol. 2015;28(2):227-236.

82. Kor DJ, et al. Effect of aspirin on development of ARDS in at-risk patients presenting to the emergency department: The LIPS-A Randomized Clinical Trial. JAMA. 2016;315(22):2406-2414.

83. Abdulnour RE, et al. Early intravascular events are associated with development of acute respiratory distress syndrome. A Substudy of the LIPS-A Clinical Trial. Am J Respir Crit Care Med. 2018;197(12):1575-1585.

84. Boomer JS, et al. Immunosuppression in patients who die of sepsis and multiple organ failure. JAMA. 2011;306(23):2594-2605.

85. Limaye AP, et al. Cytomegalovirus reactivation in critically ill immunocompetent patients. JAMA. 2008;300(4):413-422.

86. Limaye AP, et al. Effect of Ganciclovir on IL-6 levels among cytomegalovirus-seropositive adults with critical illness: A Randomized Clinical Trial. JAMA. 2017;318(8):731-740.

87. Hotchkiss RS, et al. Sepsis-induced apoptosis causes progressive profound depletion of B and CD4 $4^{+} \mathrm{T}$ lymphocytes in humans J Immunol. 2001;166(11):6952-6963.

88. Hotchkiss RS, Monneret G, Payen D. Sepsis-induced immunosuppression: from cellular dysfunctions to immunotherapy. Nat Rev Immunol. 2013;13(12):862-874.

89. Jin L, Batra S, Douda DN, Palaniyar N, Jeyaseelan S. CXCL1 contributes to host defense in polymicrobial sepsis via modulating T cell and neutrophil functions. J Immunol. 2014;193(7):3549-3558.

90. Patil NK, Guo Y, Luan L, Sherwood ER. Targeting Immune Cell Checkpoints during Sepsis. Int J Mol Sci. 2017;18(11):2413.

91. Inoue S, Bo L, Bian J, Unsinger J, Chang K, Hotchkiss RS. Dose-dependent effect of anti-CTLA-4 on survival in sepsis. Shock. 2011;36(1):38-44.

92. Chang KC, et al. Blockade of the negative co-stimulatory molecules PD-1 and CTLA-4 improves survival in primary and secondary fungal sepsis. Crit Care. 2013;17(3):R85.

93. Prieto JL, et al. Monitoring sepsis using electrical cell profiling. Lab Chip. 2016;16(22):4333-4340.

94. Meisel C, et al. Granulocyte-macrophage colony-stimulating factor to reverse sepsis-associated immunosuppression: a double-blind, randomized, placebo-controlled multicenter trial. Am J Respir Crit Care Med. 2009;180(7):640-648

95. Venet F, et al. IL-7 restores lymphocyte functions in septic patients. J Immunol. 2012;189(10):5073-5081.

96. Spitzer MH, Nolan GP. Mass cytometry: single cells, many features. Cell. 2016;165(4):780-791.

97. Morrell ED, et al. Cytometry TOF identifies alveolar macrophage subtypes in acute respiratory distress syndrome. JCI Insight 2018;3(10):e99281.

98. Nishino M, Sholl LM, Hodi FS, Hatabu H, Ramaiya NH. Anti-PD-1-related pneumonitis during cancer immunotherapy. $N$ Engl J Med. 2015;373(3):288-290. 
99. Galiacy S, et al. Keratinocyte growth factor promotes cell motility during alveolar epithelial repair in vitro. Exp Cell Res. 2003;283(2):215-229.

100. Panos RJ, Bak PM, Simonet WS, Rubin JS, Smith LJ. Intratracheal instillation of keratinocyte growth factor decreases hyperoxia-induced mortality in rats. J Clin Invest. 1995;96(4):2026-2033.

101. Ray P, et al. Inducible expression of keratinocyte growth factor (KGF) in mice inhibits lung epithelial cell death induced by hyperoxia. Proc Natl Acad Sci U S A. 2003;100(10):6098-6103.

102. Welsh DA, Summer WR, Dobard EP, Nelson S, Mason CM. Keratinocyte growth factor prevents ventilator-induced lung injury in an ex vivo rat model. Am J Respir Crit Care Med. 2000;162(3 pt 1):1081-1086.

103. McAuley DF, et al. Keratinocyte growth factor for the treatment of the acute respiratory distress syndrome (KARE): a randomised, double-blind, placebo-controlled phase 2 trial. Lancet Respir Med. 2017;5(6):484-491.

104. Millar FR, Summers C, Griffiths MJ, Toshner MR, Proudfoot AG. The pulmonary endothelium in acute respiratory distress syndrome: insights and therapeutic opportunities. Thorax. 2016;71(5):462-473.

105. Birukova AA, Tian X, Tian Y, Higginbotham K, Birukov KG. Rap-afadin axis in control of Rho signaling and endothelial barrier recovery. Mol Biol Cell. 2013;24(17):2678-2688.

106. Schnoor M, García Ponce A, Vadillo E, Pelayo R, Rossaint J, Zarbock A. Actin dynamics in the regulation of endothelial barrier functions and neutrophil recruitment during endotoxemia and sepsis. Cell Mol Life Sci. 2017;74(11):1985-1997.

107. Natarajan V, et al. Sphingosine-1-phosphate, FTY720, and sphingosine-1-phosphate receptors in the pathobiology of acute lung injury. Am J Respir Cell Mol Biol. 2013;49(1):6-17.

108. Sun X, et al. Functional promoter variants in sphingosine 1-phosphate receptor 3 associate with susceptibility to sepsis-associated acute respiratory distress syndrome. Am J Physiol Lung Cell Mol Physiol. 2013;305(7):L467-L477.

109. Thurston G, et al. Angiopoietin-1 protects the adult vasculature against plasma leakage. Nat Med. 2000;6(4):460-463.

110. Parikh SM. Dysregulation of the angiopoietin-Tie-2 axis in sepsis and ARDS. Virulence. 2013;4(6):517-524

111. David S, et al. Angiopoietin-2 may contribute to multiple organ dysfunction and death in sepsis*. Crit Care Med. 2012;40(11):3034-3041.

112. Calfee CS, Gallagher D, Abbott J, Thompson BT, Matthay MA, NHLBI ARDS Network. Plasma angiopoietin-2 in clinical acute lung injury: prognostic and pathogenetic significance. Crit Care Med. 2012;40(6):1731-1737.

113. Gutbier B, et al. Prognostic and pathogenic role of angiopoietin-1 and -2 in pneumonia. Am J Respir Crit Care Med. 2018;198(2):220-231.

114. Agrawal A, et al. Plasma angiopoietin-2 predicts the onset of acute lung injury in critically ill patients. Am J Respir Crit Care Med. 2013;187(7):736-742.

115. Sugiyama MG, et al. The Tie2-agonist Vasculotide rescues mice from influenza virus infection. Sci Rep. 2015;5:11030.

116. Han S, et al. Amelioration of sepsis by TIE2 activation-induced vascular protection. Sci Transl Med. 2016;8(335):335ra55.

117. Kümpers P, Lukasz A. The curse of angiopoietin-2 in ARDS: on stranger TI(E)des. Crit Care. 2018;22(1):44.

118. Chen W, Pendyala S, Natarajan V, Garcia JG, Jacobson JR. Endothelial cell barrier protection by simvastatin: GTPase regulation and NADPH oxidase inhibition. Am J Physiol Lung Cell Mol Physiol. 2008;295(4):L575-L583.

119. Jacobson JR, Barnard JW, Grigoryev DN, Ma SF, Tuder RM, Garcia JG. Simvastatin attenuates vascular leak and inflammation in murine inflammatory lung injury. Am J Physiol Lung Cell Mol Physiol. 2005;288(6):L1026-L1032.

120. Higuita-Castro N, Shukla VC, Mihai C, Ghadiali SN. Simvastatin treatment modulates mechanically-induced injury and inflammation in respiratory epithelial cells. Ann Biomed Eng. 2016;44(12):3632-3644.

121. National Heart, Lung, Blood Institute ARDS Clinical Trials Network, et al. Rosuvastatin for sepsis-associated acute respiratory distress syndrome. N Engl J Med. 2014;370(23):2191-2200.

122. McAuley DF, et al. Simvastatin in the acute respiratory distress syndrome. N Engl J Med. 2014;371(18):1695-1703.

123. Calfee CS, et al. Acute respiratory distress syndrome subphenotypes and differential response to simvastatin: secondary analysis of a randomised controlled trial. Lancet Respir Med. 2018;6(9):691-698.

124. Prescott HC, Calfee CS, Thompson BT, Angus DC, Liu VX. Toward smarter lumping and smarter splitting: rethinking strategies for sepsis and acute respiratory distress syndrome clinical trial design. Am J Respir Crit Care Med. 2016;194(2):147-155.

125. Reutershan J, Vollmer I, Stark S, Wagner R, Ngamsri KC, Eltzschig HK. Adenosine and inflammation: CD39 and CD73 are critical mediators in LPS-induced PMN trafficking into the lungs. FASEB J. 2009;23(2):473-482.

126. Eckle T, Grenz A, Laucher S, Eltzschig HK. A2B adenosine receptor signaling attenuates acute lung injury by enhancing alveolar fluid clearance in mice. J Clin Invest. 2008;118(10):3301-3315.

127. Kiss J, Yegutkin GG, Koskinen K, Savunen T, Jalkanen S, Salmi M. IFN-beta protects from vascular leakage via up-regulation of CD73. Eur J Immunol. 2007;37(12):3334-3338

128. Bellingan G, et al. The effect of intravenous interferon-beta-1a (FP-1201) on lung CD73 expression and on acute respiratory distress syndrome mortality: an open-label study. Lancet Respir Med. 2014;2(2):98-107.

129. Faron Pharmaceuticals Ltd. Efficacy and Safety of FP-1201-lyo (Interferon Beta-1a) in Patients Having Acute Respiratory Distress Syndrome (ARDS) (INTEREST). NIH website. https://clinicaltrials.gov/ct2/show/NCT02622724. Updated May 17, 2018. Accessed December 17, 2018

130. Rocco PR, Dos Santos C, Pelosi P. Lung parenchyma remodeling in acute respiratory distress syndrome. Minerva Anestesiol. 2009;75(12):730-740.

131. Davey A, McAuley DF, O'Kane CM. Matrix metalloproteinases in acute lung injury: mediators of injury and drivers of repair. Eur Respir J. 2011;38(4):959-970.

132.Dos Santos CC. Advances in mechanisms of repair and remodelling in acute lung injury. Intensive Care Med. 2008;34(4):619-630

133. Hogan BL, et al. Repair and regeneration of the respiratory system: complexity, plasticity, and mechanisms of lung stem cell function. Cell Stem Cell. 2014;15(2):123-138.

134. Hong KU, Reynolds SD, Watkins S, Fuchs E, Stripp BR. Basal cells are a multipotent progenitor capable of renewing the bronchial epithelium. Am J Pathol. 2004;164(2):577-588

135. Zacharias WJ, et al. Regeneration of the lung alveolus by an evolutionarily conserved epithelial progenitor. Nature. 2018;555(7695):251-255. 
136. Nabhan AN, Brownfield DG, Harbury PB, Krasnow MA, Desai TJ. Single-cell Wnt signaling niches maintain stemness of alveolar type 2 cells. Science. 2018;359(6380):1118-1123.

137. Laffey JG, Matthay MA. Fifty Years of Research in ARDS. Cell-based Therapy for Acute Respiratory Distress Syndrome. Biology and Potential Therapeutic Value. Am J Respir Crit Care Med. 2017;196(3):266-273.

138. Wilson JG, et al. Mesenchymal stem (stromal) cells for treatment of ARDS: a phase 1 clinical trial. Lancet Respir Med. 2015;3(1):24-32.

139. Matthay MA, et al. Treatment with allogeneic mesenchymal stromal cells for moderate to severe acute respiratory distress syndrome (START study): a randomised phase 2a safety trial [published online ahead of print November 16, 2018]. Lancet Respir Med. https://doi.org/10.1016/S2213-2600(18)30418-1.

140. Ortega-Gómez A, Perretti M, Soehnlein O. Resolution of inflammation: an integrated view. EMBO Mol Med. 2013;5(5):661-674.

141. Voll RE, Herrmann M, Roth EA, Stach C, Kalden JR, Girkontaite I. Immunosuppressive effects of apoptotic cells. Nature. 1997;390(6658):350-351.

142. Byrne A, Reen DJ. Lipopolysaccharide induces rapid production of IL-10 by monocytes in the presence of apoptotic neutrophils. J Immunol. 2002;168(4):1968-1977.

143. Zemans RL, Henson PM, Henson JE, Janssen WJ. Conceptual approaches to lung injury and repair. Ann Am Thorac Soc. 2015;12(suppl 1):S9-15.

144. Zemans RL, Matthay MA. Bench-to-bedside review: the role of the alveolar epithelium in the resolution of pulmonary edema in acute lung injury. Crit Care. 2004;8(6):469-477.

145. Mutlu GM, Sznajder JI. Mechanisms of pulmonary edema clearance. Am J Physiol Lung Cell Mol Physiol. 2005;289(5):L685-L695.

146. Peteranderl C, et al. Macrophage-epithelial paracrine crosstalk inhibits lung edema clearance during influenza infection. J Clin Invest. 2016;126(4):1566-1580.

147. Ware LB, Matthay MA. Alveolar fluid clearance is impaired in the majority of patients with acute lung injury and the acute respiratory distress syndrome. Am J Respir Crit Care Med. 2001;163(6):1376-1383.

148. National Heart, Lung, Blood Institute Acute Respiratory Distress Syndrome (ARDS) Clinical Trials Network, et al. Randomized, placebo-controlled clinical trial of an aerosolized $\beta_{2}$-agonist for treatment of acute lung injury. Am J Respir Crit Care Med. 2011;184(5):561-568

149. Gao Smith F, et al. Effect of intravenous $\beta_{2}$-agonist treatment on clinical outcomes in acute respiratory distress syndrome (BALTI-2): a multicentre, randomised controlled trial. Lancet. 2012;379(9812):229-235.

150. Festic E, et al. Randomized Clinical Trial of a Combination of an Inhaled Corticosteroid and Beta Agonist in Patients at Risk of Developing the Acute Respiratory Distress Syndrome. Crit Care Med. 2017;45(5):798-805.

151. Aggarwal NR, King LS, D'Alessio FR. Diverse macrophage populations mediate acute lung inflammation and resolution. Am J Physiol Lung Cell Mol Physiol. 2014;306(8):L709-L725.

152. D'Alessio FR, et al. CD4 ${ }^{+} \mathrm{CD} 25^{+} \mathrm{Foxp}^{+}$Tregs resolve experimental lung injury in mice and are present in humans with acute lung injury. J Clin Invest. 2009;119(10):2898-2913.

153. Levy BD, Serhan CN. Resolution of acute inflammation in the lung. Annu Rev Physiol. 2014;76:467-492.

154. Herold S, Mayer K, Lohmeyer J. Acute lung injury: how macrophages orchestrate resolution of inflammation and tissue repair Front Immunol. 2011;2:65.

155. Seki $\mathrm{H}$, et al. The anti-inflammatory and proresolving mediator resolvin E1 protects mice from bacterial pneumonia and acute lung injury. J Immunol. 2010;184(2):836-843.

156. Dalli J, et al. Human Sepsis Eicosanoid and Proresolving Lipid Mediator Temporal Profiles: Correlations With Survival and Clinical Outcomes. Crit Care Med. 2017;45(1):58-68. 